\title{
ANALYSIS OF RADAR ABSORBING FSS ON FOLDCORES AND HONEYCOMBS
}

\author{
J.J.P. VAN ES ${ }^{*}$, A. HULZINGA ${ }^{*}$, P. TENSEN ${ }^{*}$, H. SCHIPPERS ${ }^{*}$, \\ R.M.H. HEIJMANS ${ }^{\dagger}$, M.J.G. JOURNEE ${ }^{\dagger}$ \\ * Netherlands Aerospace Centre (NLR) \\ Voorsterweg 31, 8316 PR Marknesse, The Netherlands \\ e-mail: Jan-Joris.van.Es@nlr.nl,www.nlr.nl \\ ${ }^{\dagger}$ Teijin Aramid B.V. \\ Velperweg 76, 6824 BM Arnhem, The Netherlands \\ email: Ruud.Heijmans@Teijinaramid.com,www.teijinaramid.com
}

Key words: Computational Methods, Metamaterials, Radar absorption

\begin{abstract}
The objective of the paper is to investigate the radar absorption of honeycombs and foldcores with printed conductive patterns. These structures can be manufactured by first printing conductive Frequency Selective Surfaces (FSS) on planar substrates, which then can be used to shape foldcores and honeycombs by means of specific manufacturing technologies. Foldcores can be considered as intermediate shapes between planar sheets (where the printed patterns are perpendicular to the impinging radar waves) and honeycombs (where the printed patterns are parallel to the impinging radar wave). It is shown that the radar absorbing properties of the design strongly depend on the electrical conductivity of the paint, the size of the printed patterns and the orientation of the printed patterns with respect to the impinging wave. It is shown that a planar FSS has a maximum absorption of 50\%, while foldcores and honeycombs may obtain a higher absorption due to the fact that the patterns are orientated under an angle with respect to the propagation direction of the wave.
\end{abstract}

\section{INTRODUCTION}

This research deals with the development of advanced radar absorbing structures with artificial structured metamaterials. These materials can be realised by printing arrays of electrically conducting patterns (such as Split-Ring resonators) on non-conducting sheets. The goal of the conducting patterns is to modify the EM properties of the structure and to influence the way incident EM waves are reflected and transmitted. The structure can be made lossy, i.e. absorbing EM energy, by applying the right patterns and right electric conductivity.

The challenge is two-fold. There is the question of how the conducting patterns should look and what size they should have in order to obtain the desired EM properties. On the other hand there is the question of actually fabricating such a structure. Screen printing and inkjet printing with electrically conducting ink appear valid options to produce the desired shapes. The substrates can be FR4 glass epoxy, aramid honeycomb papers, or other non-conducting sheets. Once successful the printed 2D sheets can be used to create 3D structures such as honeycombs and foldcores with tailored EM properties. 
The subject of tailoring the EM properties of 3D arrangements of electrically conducting patterns is not new and has been the topic of earlier research [1,2,3]. In these earlier publications the emphasis is on achieving particular values of permittivity and permeability. In the current work we focus on the radar absorbing properties of the materials and the optimisation of the conductivity to maximise absorption.

\section{GEOMETRIES}

The electrically conducting patterns in this research are circular rings and split-ring resonators (SRR). The conducting patterns are repeated in a regular way to form an infinite sheet. Depending on the orientation of the pattern in the unit cell and the repetition vector, the sheet can be an infinitely thin FSS, a honeycomb or a foldcore (Figure 1).

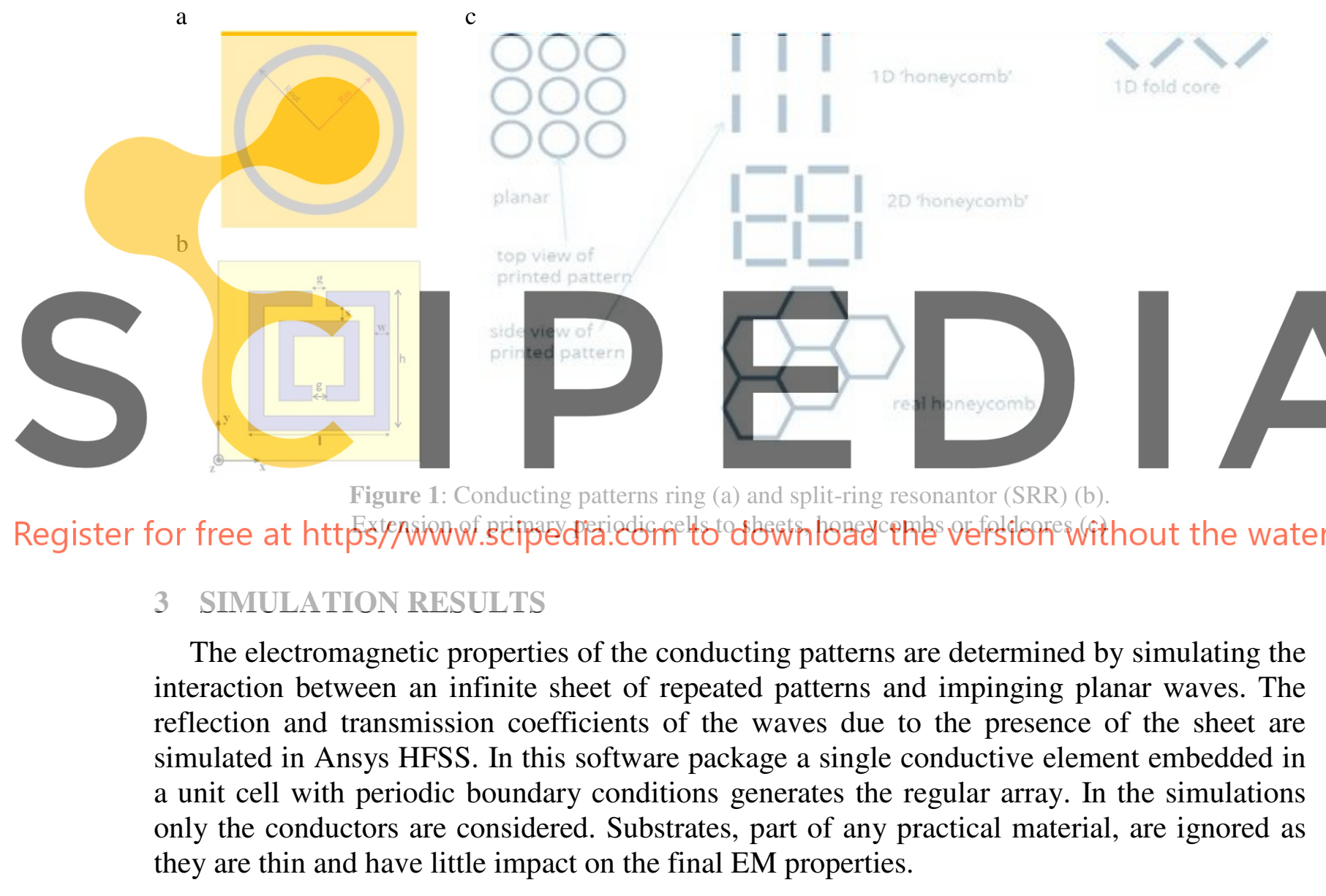

\subsection{Size}

The size and the exact shape of the conducting patterns determine the resonant behaviour. In the current work the conducting patterns are designed for maximum effect in X-band (8.212.4 GHz). The SRR has a $4 \mathrm{~mm}$ width (l), $3 \mathrm{~mm}$ height (h), $0.5 \mathrm{~mm}$ spacing between the rings (s), $0.5 \mathrm{~mm}$ gap $(\mathrm{g})$ and $0.3 \mathrm{~mm}$ trace width $(\mathrm{w})$. The conducting rings have an inner radius of $4.2 \mathrm{~mm}$ and an outer radius of $4.8 \mathrm{~mm}$ (Figure 1 a and b). 


\subsection{Electric conductivity}

The impinging EM waves induce currents in the conducting patterns. These currents experience Ohmic losses which gives rise to the radar absorbing effect. The rings need to have a specific conductivity for optimum absorption. If the electric conductivity is too high currents will be induced in the patterns, but the currents will experience little losses. If the conductivity is too low little currents will be induced and the waves will propagate unobstructed. The absorption, $A$, is calculated from the (complex-valued) transmission, $T$, and reflection, $R$, coefficients in the HFSS simulation results as

$$
A=1-R R^{*}-T T^{*}
$$

To illustrate the importance of the value of the conductivity, the reflection and transmission coefficients have been computed for perfectly conducting rings in a planar sheet FSS. The results are shown in Figure 2. Obviously the rings are resonant at $12 \mathrm{GHz}$. For these perfectly conducting rings there is no absorption. Next the absorption coefficient is computed in relation to the conductivity of these rings. From Figure 3 it is concluded that the amount of absorption strongly depends on the conductivity. The conductivity of the rings can be quantified in different ways. It can be given as the sheet resistivity $\left(\mathrm{R}_{\mathrm{S}}\right)$, the parallel resistance $\left(\mathrm{R}_{\|}\right)$of two half rings when measured across with two probes or as the resistance of the whole ring when it would be interrupted by an infinitely narrow slit $\left(\mathrm{R}=4 \mathrm{R}_{\|}\right) . \mathrm{R}_{\mathrm{s}}$ and $\mathrm{R}_{\|}$relate as

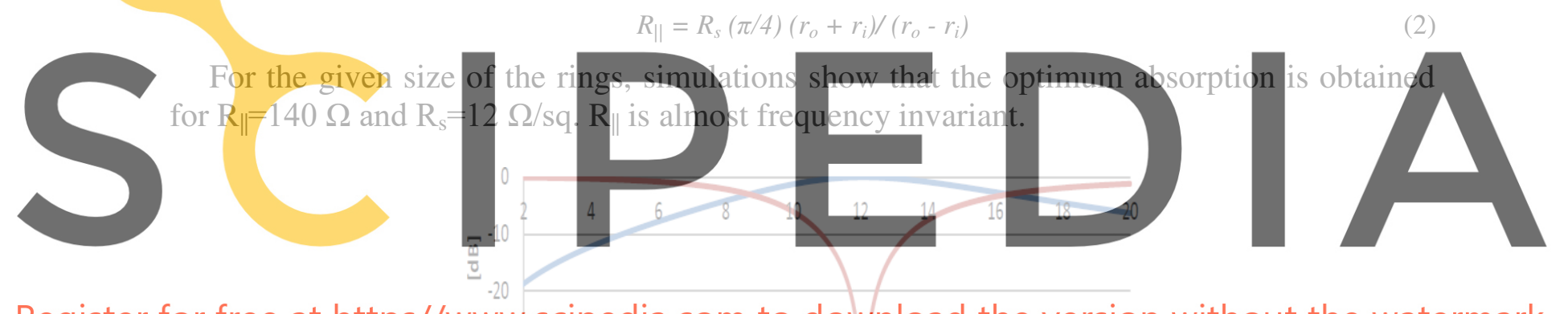

Register for free at https//www.scipedia.com to download the version without the watermark

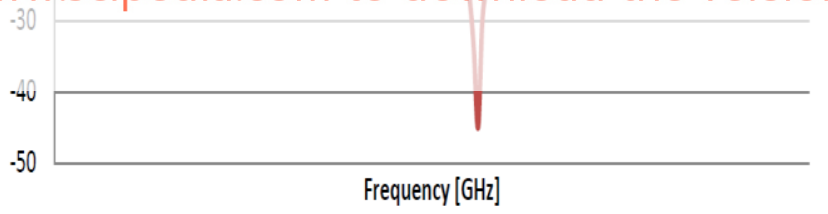

- reflection - transmission

Figure 2: Reflection and transmission coefficient of planar sheet with perfectly conducting rings; (inner radius : $4.2 \mathrm{~mm}$, outer radius $4.8 \mathrm{~mm}$, cell size $11 \mathrm{~mm}$ ) 


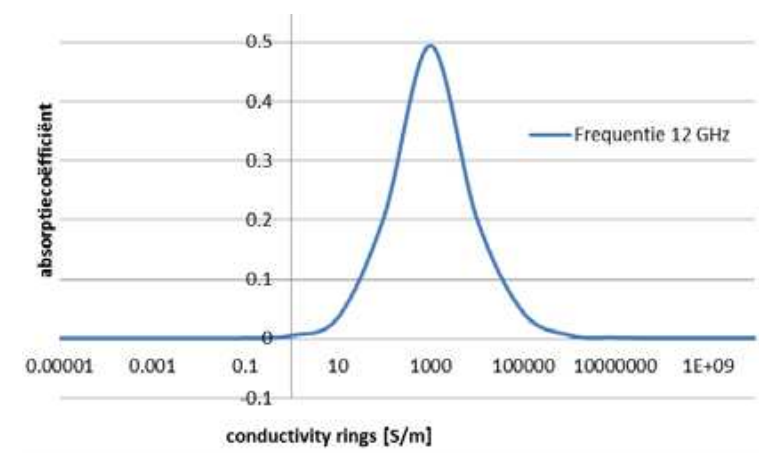

Figure 3: Absorption coefficient of planar sheet with conducting rings at $12 \mathrm{GHz}$ as function of the bulk conductivity of the rings

\subsection{Orientation}

For planar sheets of conducting patterns with zero thickness the maximum absorption is found to be $0.5(-3 \mathrm{~dB})$ (Figure 3). Using transmission line theory, this case can be modelled as a lumped impedance $Z_{L}$ in parallel with the wave impedance of the propagation medium $Z_{0}$. In this case the reflection and transmission coefficients are given as

$$
R=-Z_{0} /\left(Z_{0}+2 Z_{L}\right)
$$

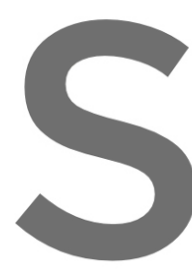

such that indeed the abso

patterns are oriented dut

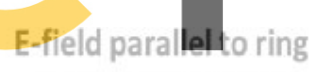

$$
\text { theta }=0 \text { degrees }
$$

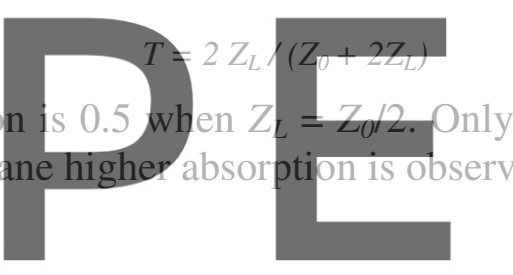

(n)

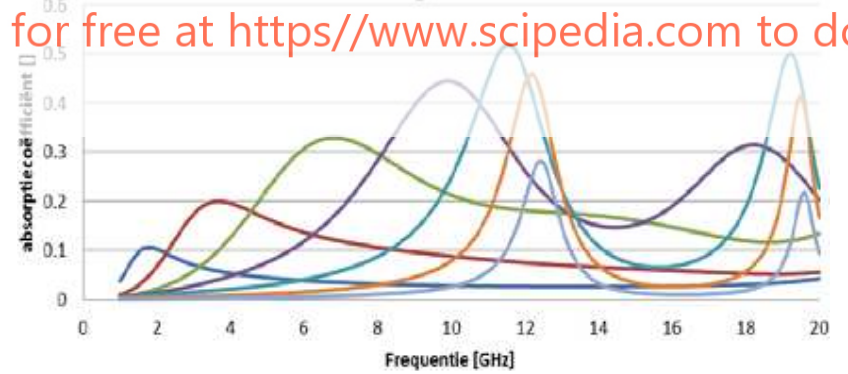

$-0.1 \mathrm{~s} / \mathrm{m}-1 \mathrm{~s} / \mathrm{m}-10 \mathrm{~s} / \mathrm{m}-100 \mathrm{~s} / \mathrm{m}-1 \mathrm{E} 3 \mathrm{~s} / \mathrm{m}-1 \mathrm{E} 4 \mathrm{~s} / \mathrm{m}-1 \mathrm{Es} / \mathrm{m}$

Figure 4: Absorption of 1D honeycomb with rings for different conductivities;

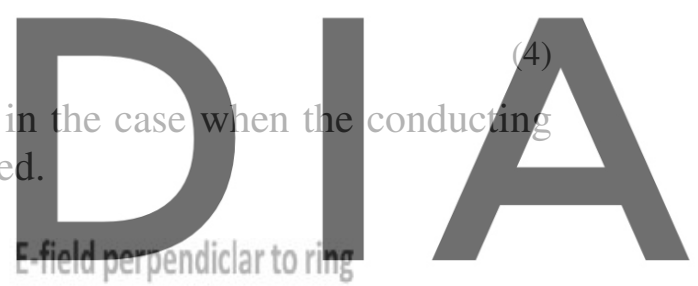

theta $=0$ degrees

Register for free at https//www.scipedia.com to download the version without the watermark direction E-field parallel to the rings (left), direction of E-field perpendicular to the rings (right) 


\section{E-field paralllel to ring theta $=0$ degrees}

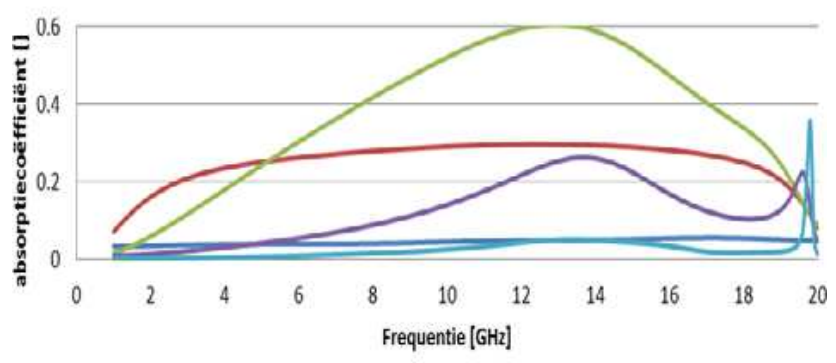
$-10-100-1000-1.00 \mathrm{E}+04-1.00 \mathrm{E}+05$

Figure 5: Absorption of 1D foldcore with rings for different conductivities; direction E-field parallel to the rings (left), direction of E-field perpendicular to the rings (right)
E-field perpendicular to ring theta $=0$ degrees

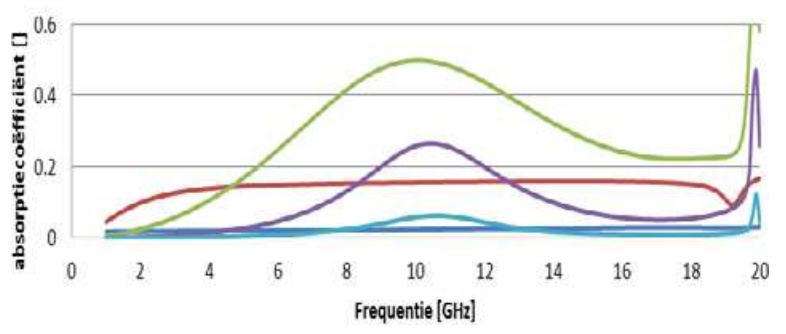

$$
-10-100-1000-1.00 E 104-1.00 E 105
$$

The orientation of the conducting patterns with respect to the E-field of the impinging influences the magnitude of the induced current in the patterns and thus the absorption. The induced current and the losses are largest with the conductors parallel to the electric field vector. This effect is nicely illustrated in a 1D honeycomb with conducting rings (Figure 4). The different colours in this figure indicate different values of the conductivity. Generally the variation in absorption is smaller in foldcores, because the patterns are oriented in two or

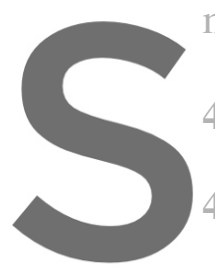
more angles out of plane (Figure 5 ).

4 FABRICATION OF TEST SAMPLES

4.1 Foldcores

Samples of foldcores with conducting pat
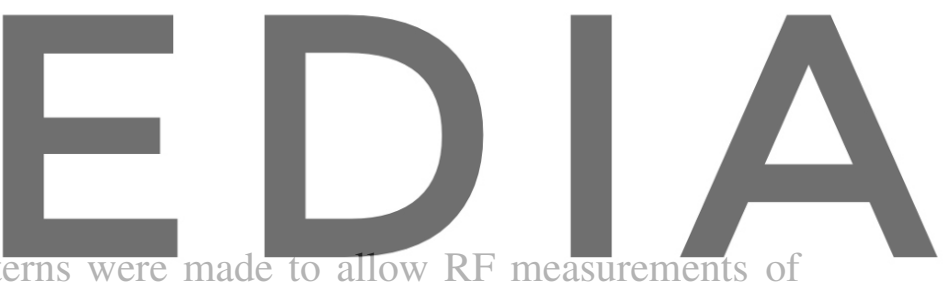

the absorbing properties (Figure 6). First the conducting patterns were printed on $54 \mathrm{~g} / \mathrm{m} 2$

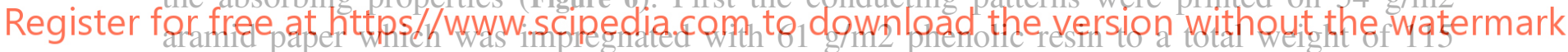

$\mathrm{g} / \mathrm{m} 2$. The patterns were applied with 120 mesh screen printing of electrically conducting ink.

The conductivity of the patterns was tuned to the desired value by biending a low and a highconductivity ink to the desired value. In some cases the conductivity of the patterns was increased by printing multiple (two) layers of the same ink.

After printing and drying, the paper was folded into the desired foldcore shape or honeycomb shape. Finally the foldcores were cured for about 1 hour at $150^{\circ} \mathrm{C}$ to secure the shape. The curing also improved the conductivity of the printed patterns. The exact curing duration and temperature were used to optimise the conductivity.

Finally, the conductivity of the conducting rings was characterized through measurement of the resistance $\left(R_{\|}\right)$between the two sides of the rings using a two-probe method. The ring resistance typically shows a variation in the order of $10 \%$. The foldcore samples have a size of about $25 \times 16 \mathrm{~cm}$ and a height of $2 \mathrm{~cm}$. 


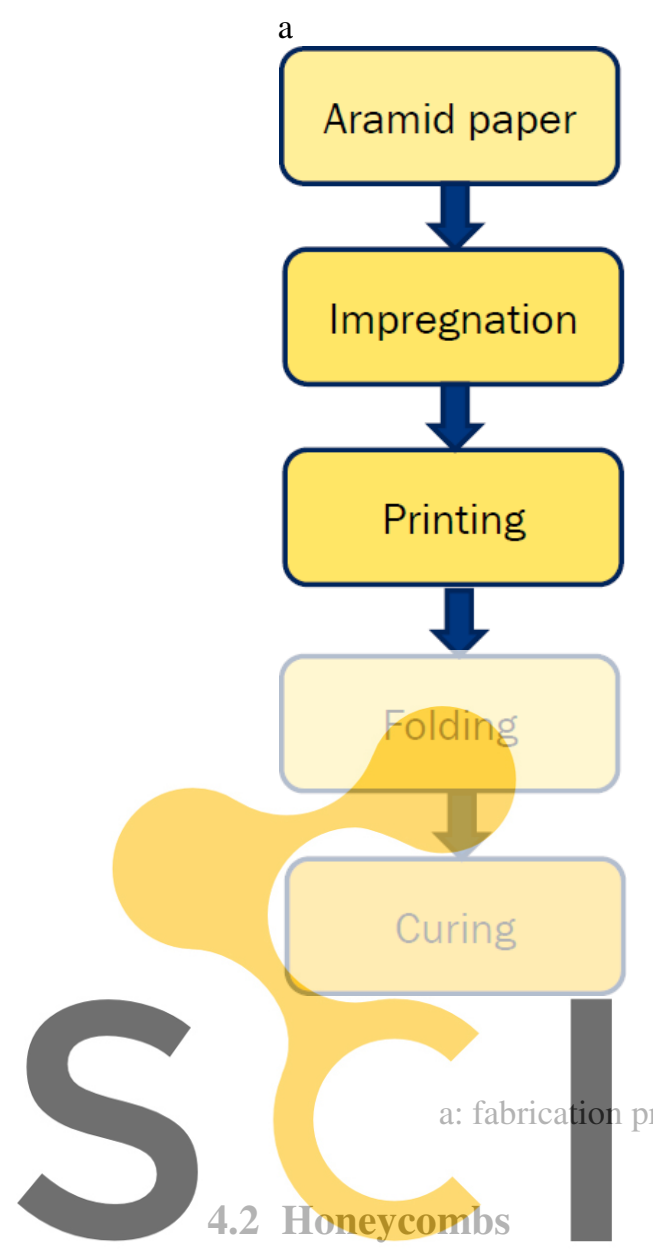

b

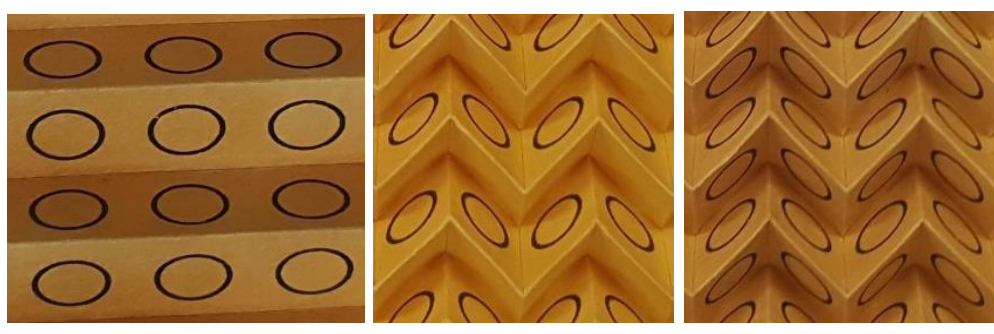

$\mathrm{c}$

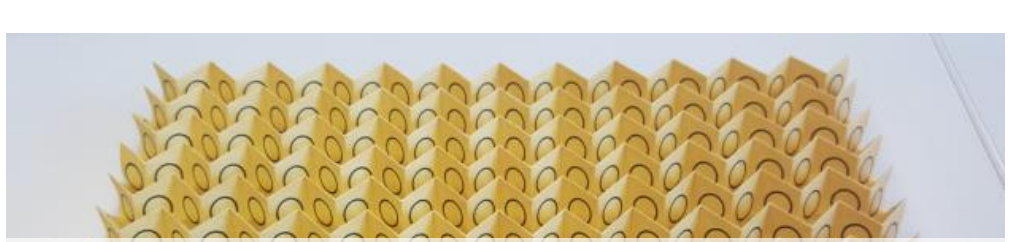

Honeycomb samples with SRRs were fabricated in a similar way as the foldcore samples.

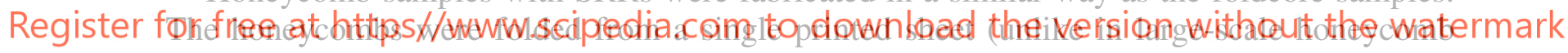
fabrication). The samples measure $7.5 \mathrm{~cm} \times 4 \mathrm{~cm}$ and have a thickness of $0.5 \mathrm{~cm}$. Samples with carbon ink (236 Ohm/sq) and silver ink (0.05 Ohm/sq) were made (Figure 7).
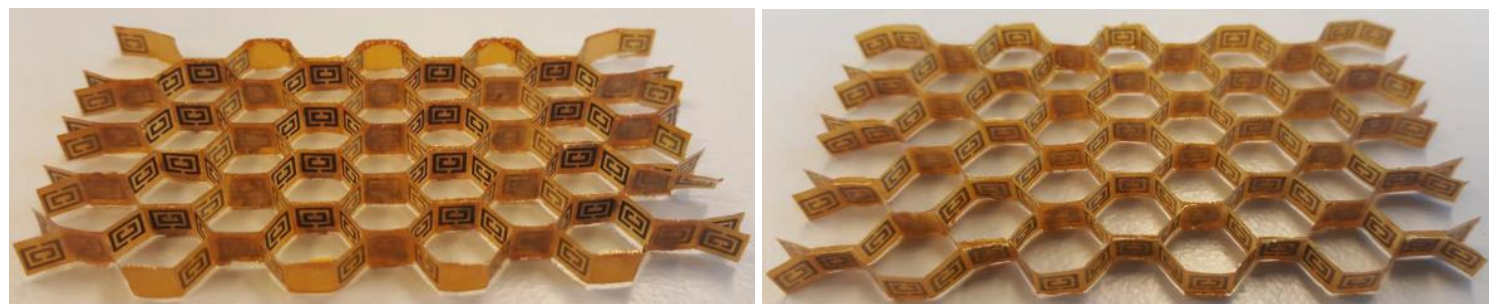

Figure 7: Honeycomb samples with SRR rings in carbon (left) and silver ink (right)

\section{MEASUREMENT RESULTS}

Both foldcore and honeycomb samples were measured in the frequency range between 1 and $20 \mathrm{GHz}$ in free-space reflection-transmission setups to determine the absorption. For these measurements six foldcore samples were combined in a single panel of $50 \mathrm{~cm} \times 50 \mathrm{~cm}$ 
on a Styrofoam substrate. The honeycomb samples were measured individually, placed in an aperture in an absorbing plate (Figure 8). All measurements were performed with normal incidence.

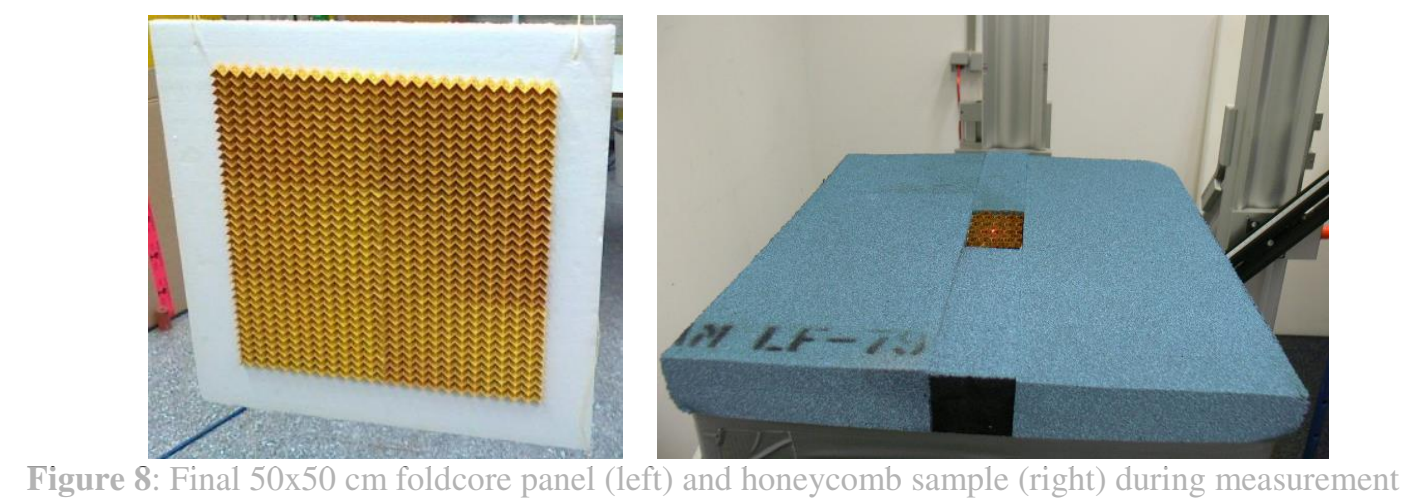

\subsection{Foldcores}

Foldcore samples with rings with two different conductivities (120 $\Omega / \mathrm{sq}$ and $12 \Omega / \mathrm{sq})$ were fabricated and measured (Figure 9). The conductivity of $12 \Omega /$ sq is approximately optimal and yields an absorption of 0.55 ; nearly the simulated value of 0.6 . The maximum is found for $\mathrm{HH}$ polarization where the E-field vector is best aligned with the conducting patterns. The
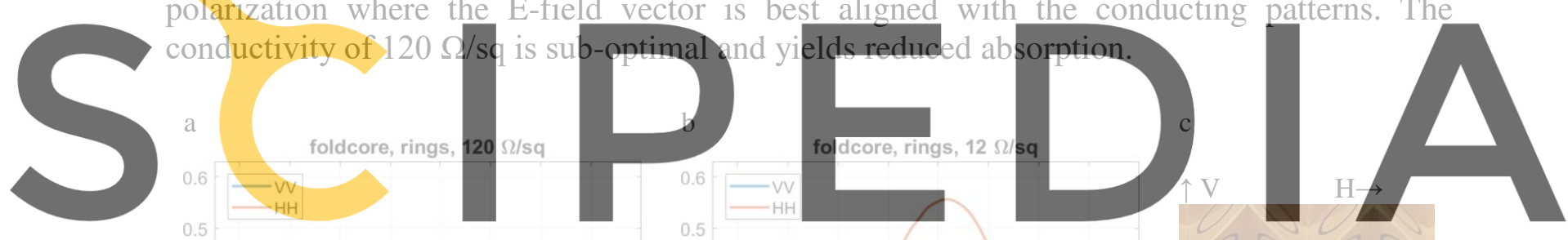

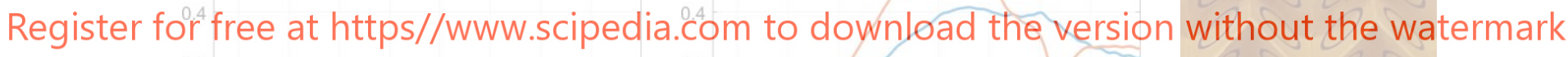
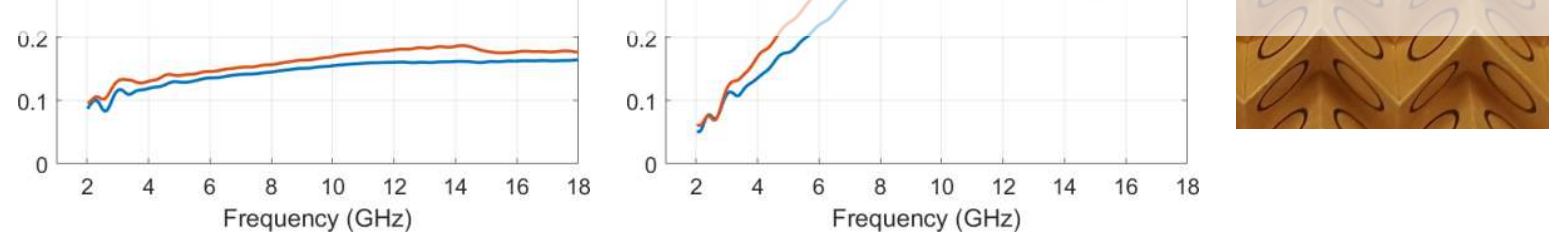

Figure 9: Measured absorption in (a) foldcore with rings Rs $=120 \Omega /$ sq and (b) foldcore with rings $\mathrm{Rs}=12 \Omega / \mathrm{sq}$. The polarization is indicated in (c).

\subsection{Honeycombs}

The honeycomb measurement results show the importance of the conductivity of the patterns (Figure 10). The carbon ink $(236 \Omega / \mathrm{sq})$ has too low conductivity to yield a significant effect and the radar wave passes through the sample without much interaction. The high conductivity silver ink $(0.05 \Omega / \mathrm{sq})$ makes visible the double resonant structure of the SRR. It has a maximum absorption of $0.67(-1.7 \mathrm{~dB})$ with $\mathrm{HH}$ polarization at $9.65 \mathrm{GHz}$. 

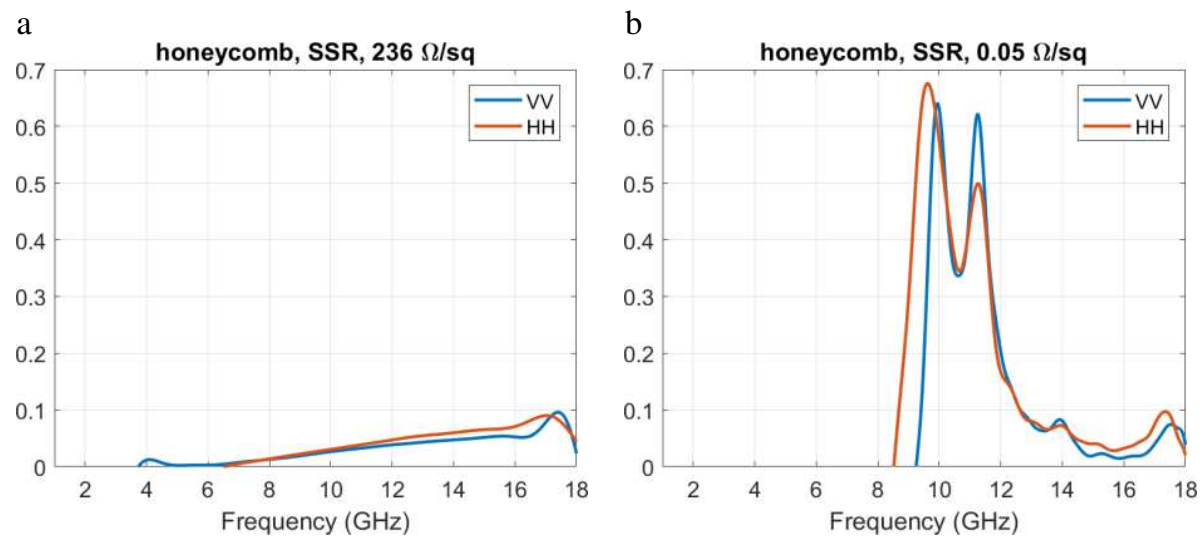

$\mathrm{c}$

Figure 10: Measured absorption (A) of honeycomb with SRR in carbon ink (236 $\Omega / \mathrm{sq}$ ) (a) and in silver ink $(0.05 \Omega / \mathrm{sq})(\mathrm{b})$. The polarization is indicated in $(\mathrm{c})$.

\section{CONCLUSIONS}

This paper investigates radar absorbing structures based on foldcore and honeycomb sandwich cores with printed electrically conducting patterns. RF absorption is created by Ohmic losses of currents induced in the patterns. To achieve optimal absorption it is necessary that the E-field of the incident wave is parallel to the conducting pattern and that the conductivity has a specific value. Honeycomb and foldcore samples with patterns with the desired conductivity

losses. The lossy foldco lightweight structures with tailored EM patterns consisting of SRRs and rings resonan further optimise the EM properties at X-band or a
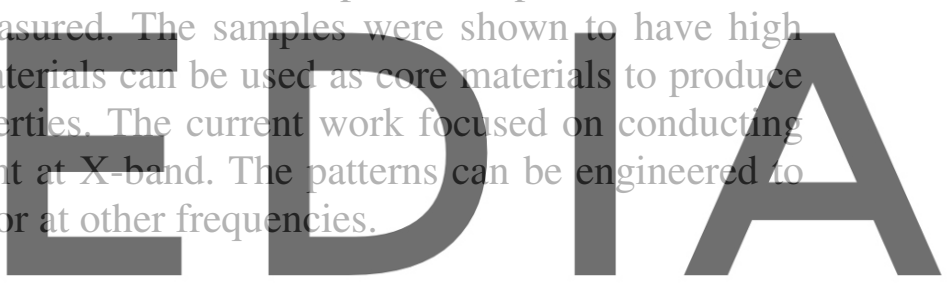

7 ACKNOWLEDGEMENTS

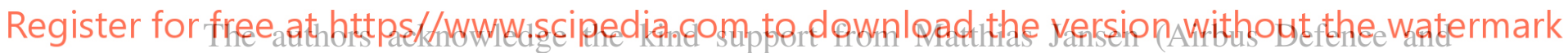

Space) in the measurement of the honeycomb samples.

\section{REFERENCES}

[1] Diaz, Rodolfo E. Material with artificial dielectric constant United States Patent 5662982, 2 Sept 1997.

[2] Houck, Andrew A.; Brock, Jeffrey B.; Chuang, Isaac L. Experimental observations of a left-handed material that obeys Snell's law Phys. Rev. Lett. 90(13), 137401, 2003.

[3] Ekmekci E.; Turhan-Sayan G. Comparative investigation of resonance characteristics and electrical size of the double-sided SRR, BC-SRR and conventional SRR type metamaterials for varying substrate parameters Progress In Electromagnetics Research B, Vol. 12, 35-62, 2009. 